\title{
CIENCIA\&SALUD
}

\section{Abordaje radiológico en pacientes con sospecha de aneurisma}

aórtico torácico.

Radiologic approach in patients with suspected thoracic aortic

aneurism

Diego Cerdas SotoVerónica Fernández Angulo²María José Ureña Vargas³Reiner Sánchez

Carballo ${ }^{4}$ Gabriel Kivers Bruno ${ }^{5}$

1 Médico general Caja costarricense de seguro social, San José Costa Rica.

2, 3, 4 y 5 Médico general. Trabajador independiente, San José Costa Rica.

Contacto: dcerdas_77@hotmail.com

\section{Cómo citar:}

Cerdas Soto, D., Fernández Angulo, V. ., Ureña Vargas, M. J. ., Sánchez Carballo, R. ., \& Kivers Bruno, G. . Abordaje radiológico en pacientes con sospecha de aneurisma aórtico torácico. Revista Ciencia Y Salud, 6(1), Pág. 15-24. https://doi. org/10.34192/cienciaysalud. v6i1.349

Recibido: 07/Feb/2021

Aceptado: 02/Feb2022

Publicado: 14/Feb/2022

\section{RESUMEN}

El aneurisma de Aorta, independientemente de la localización, es una situación de urgencia en la cual se necesita una rápida captación de la patología y una decisión pronta del tratamiento. Por esta razón, es necesaria una correcta interpretación de signos y síntomas así como de estudios de imagen, los cuales serán abordados en el presente artículo así como las generalidades necesarias para poder actuar a tiempo. Se hará una revisión de cómo interpretar de manera adecuada la patología en diferentes estudios de imagen y se explicará de manera breve la población de riesgo.

Se abordará la patología torácica, esto debido a la diferencia en cantidad de bibliografía cuando se compara con el aneurisma abdominal.

Si bien es cierto no es la patología más común, según se discutirá, sí es importante a nivel estadístico conocer la cantidad de casos que se presentan en un servicio de salud primario ya que la mortalidad va a depender del abordaje que se realice y el tiempo en el que se tomen las decisiones.

Palabras Clave: Aneurisma, Aorta, Imagen

\section{ABSTRACT}

Aortic aneurysm, regardless of its location, is an emergency situation in which a rapid uptake of the pathology and a prompt decision on treatment are needed. Therefore, a correct interpretation of signs and symptoms as well as imaging studies is necessary, which will be addressed in this article with the necessary generalities to be able to act on time, a review will be made of how to properly interpret the pathology in different imaging studies and the population at risk will be briefly explained.

Thoracic pathology will be addressed, this due to the difference in number of studies when compared with abdominal aneurysm.

Although it is true it is not the most common pathology, as will be discussed, if it is statistically important to know the number of cases that occur in a primary health service since mortality will depend on the approach that is carried out and the time on which decisions are made.

Keywords: Aneurysm, Aorta, Image 


\section{INTRODUCCIÓN}

La palabra aneurisma proviene del griego aneurysma, que significa dilatación, esto a pesar de no ser esencial, brinda una pista de que se trata de una patología ya conocida de hace siglos, sin embargo hasta el día de hoy es un tema temido por profesionales del área de salud debido al grado de complejidad y que el resultado final para el paciente puede ser mortal; sin embargo se determinará cuáles pacientes son los que se se deben priorizar. A pesar de que no entraremos en detalle en la parte de tratamiento, sí nos adentraremos en el área del abordaje para poder diferenciar y actuar a tiempo.

\section{Artículos:}

- New England Journal of Medicine

- Revista Chilena de Cirugía

- Ventricular/Vascular Coupling: Clinical, Physiological and Engineering

- Guidelines for the diagnosis and management of patients with Thoracic Aortic Disease

\section{Libros:}

- Learning Radiology, Recognizing the basics, William Herring

- Diagnostic and Surgical Imaging Anatomy, Federle

- Atlas de Anatomía Humana por técnicas de imagen, Weir - Abrahams

- Patología estructural y funcional, Kumar V, Abbas AK, Aster JC. Robbins y Cotran

Páginas Web:

- Medigraphic

- UptoDate

- Colegio Argentino de Cardiología

- Elsevier

\section{MÉTODO:}

Revisión de cada texto de forma individual, con extracción de material relevante para el presente artículo.

\section{Criterios de Selección:}

- Artículos Recientes

- Información respaldada por 15 o más bibliografías

- Artículos que no hayan sido refutados mediante otros atículos de la misma especialidad o por estudios

- Libros en sus últimas ediciones

\section{DESARROLLO:}

En términos generales, un aneurisma se puede definir como una dilatación permanente y localizada de una arteria que tenga, al menos, un 50\% de aumento en el diámetro comparado con el diámetro normal de la arteria en cuestion. 1 Ya con esto se puede dar una idea de lo que está pasando en dicho paciente, en la arteria que esté siendo afectada, pero para guiarse no solo se tiene que saber la definición sino valerse del paciente.

Se puede profundizar un poco en la fisiopatología de un aneurisma sabiendo que puede pasar en cualquier arteria del cuerpo, aunque si bien es cierto no hay literatura que revise la estadística de las localizaciones más comunes, generalmente, los aneurismas más relevantes son cerebrales o aórticos; ahora bien, una de las formas mas sencillas de explicarlo es que el aneurisma no es la presencia de una simple dilatación de la 
pared arterial sino también un aumento simultáneo de su masa parietal por un intenso proceso inflamatorio localizado que, al comprometer tanto la síntesis como la degradación de la matriz proteica de la pared aórtica, formada básicamente por colágeno y elastina, incrementa la producción del colágeno tipo III, en desmedro del tipo I y reduce la proporción de elastina llevando a la fragmentación de sus unidades laminares, lo que hace disminuir tanto la capacidad de recuperación del diámetro arterial como el tono arterial, a semejanza de lo que ocurre en las arterias envejecidas. 2

Los aneurismas tienen una clasificación mayor o una descripción universal, pueden ser: aneurisma verdadero (fusiforme o sacular) y aneurisma falso (pseudoaneurisma). Se habla de un aneurisma verdadero cuando están afectadas las tres paredes arteriales (íntima, media y adventicia). En el subtipo fusiforme hay una dilatación simétrica de toda la circunferencia, mientras que en el sacular hay una dilatación más localizada, semejante a una evaginación. 3

\section{Fig 1. Tipos de Aneurismas}

El aneurisma torácico, el cual es el tema principal del artículo, se va a presentar en el $10 \%$ de los casos y es menos frecuente que el aneurisma de aorta abdominal.4 A pesar de los porcentajes de presentación mencionados, no es despreciable y por el contrario puede ser relativamente común si se tiene en cuenta a la población con la que se está tratando, el aumento del sedentarismo y las cardiopatías en general.

Como ya ha sido estudiado por muchos autores, para que una dilatación sea considerada aneurisma se tiene que tener el conocimiento de los rangos o diámetros normales de la aorta. Esta al tener diferentes porciones y al dar diversas ramas, es

lógico que tenga diámetros variables, sin

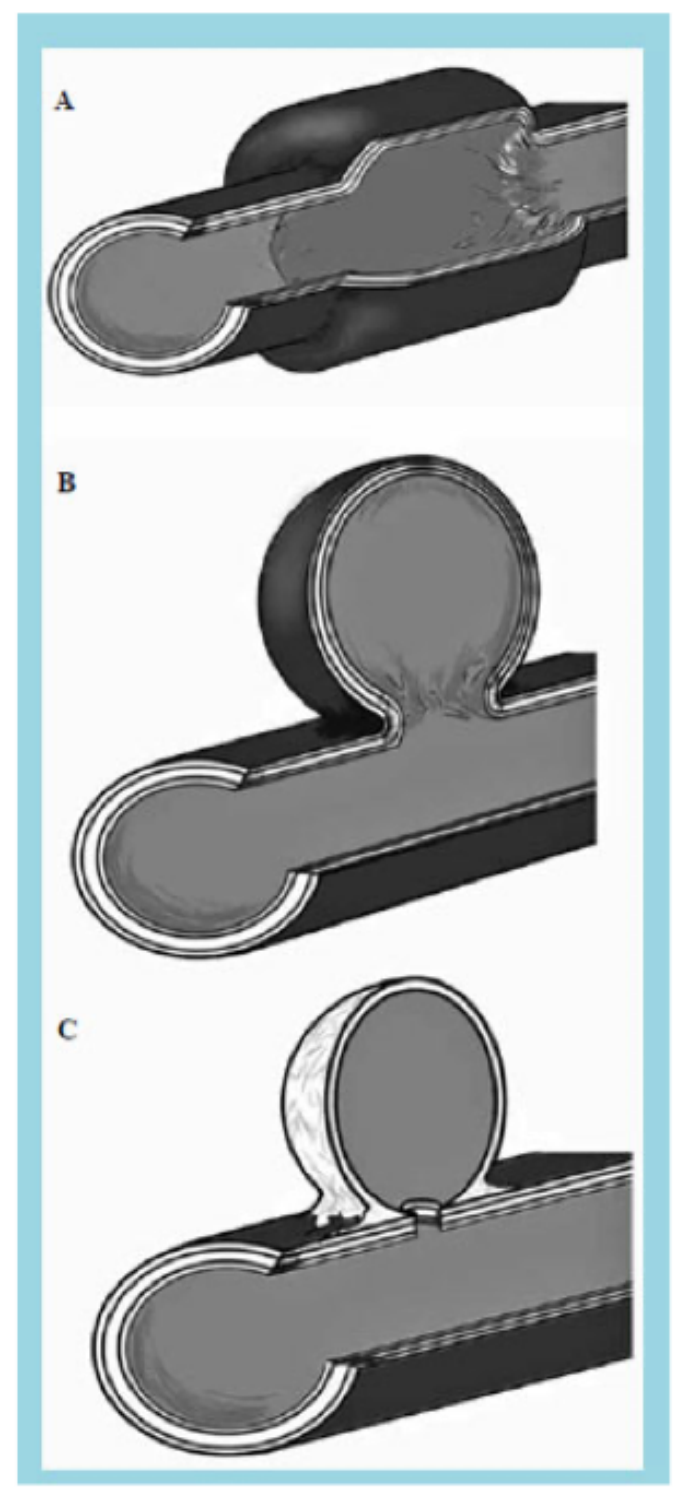




\section{CIENCIA\&SALUD}

embargo nos centraremos en la porción torácica

Tabla 1. Rangos normales de diámetro de la aorta torácica en adultos (cm)5.

\begin{tabular}{|c|c|}
\hline Aorta ascendente (hombre, mujer) & 2,86 \\
\hline Arco aórtico (hombre) & 3,63 a 3,91 \\
\hline Arco aórtico (mujer) & 3,50 a 3,72 \\
\hline Aorta descendente proximal (hombre) & 2,39 a 2,98 \\
\hline Aorta descendente proximal (mujer) & 2,45 a 2,64 \\
\hline Aorta desdendente distal (hombre) & 2,43 a 2,69 \\
\hline Aorta desdendente distal (mujer) & 2,40 a 2,44 \\
\hline
\end{tabular}

En general, los aneurismas de aorta torácicos se relacionan más frecuentemente a hipertensión arterial y otros síndromes genéticos 6 . Debido a esto, se debe tener en cuenta a la población más susceptible a esta patología, ya sea por edad, patologías de fondo, o comorbilidades.

Se han relacionado diversas enfermedades o síndromes a la dilatación aórtica, como por ejemplo:

Tabla 2. Patologías asociadas a dilatación aórtica 7

Como se mencionó previamente, se ha visto un incremento de enfermedades cardiovasculares con respecto a años previos debido a los avances médicos y aumento en la expectativa de vida.

En la siguiente tabla se evidencia en un hospital de São Paulo, Brasil la incidencia en aumento de enfermedades de esta índole:

1. La degeneración cística idiopática de la capa media de la pared de la aorta.

2. Degenerativos:

a. Aterosclerosis

b. Fibrodisplasia

3. Enfermedades genéticas que se asocian con alteraciones en la colágena:

a. Síndrome de Marfan

b. Síndrome de Ehlers-Danlos

c. La enfermedad familiar dilatante de la aorta

4. La disección de la aorta que produce aneurisma de la aorta

5. La enfermedad de la válvula aórtica asociada con dilatación de la aorta

a. Aorta bivalva

b. Aorta monovalva

6. Infección:

a. Aneurismas bacterianos (Micóticos)

b. Aneurismas sifilíticos

7. Enfermedades asociadas a arteritis:

a. Lupus

b. Arteritis de células grandes

c. Takayasu

8. Congénitos

9. Trauma

10. Pseudoaneurismas 
Tabla 3. La incidencia de enfermedades de la aorta ha ido aumentando con el paso de los años en el Servicio de Cirugía Cardíaca de la Universidad de São Paulo. Cortesía del Dr. Enio Buffolo, Universidad de São Paulo, São Paulo Brasil. 8

\section{Enfermedades de la aorta}

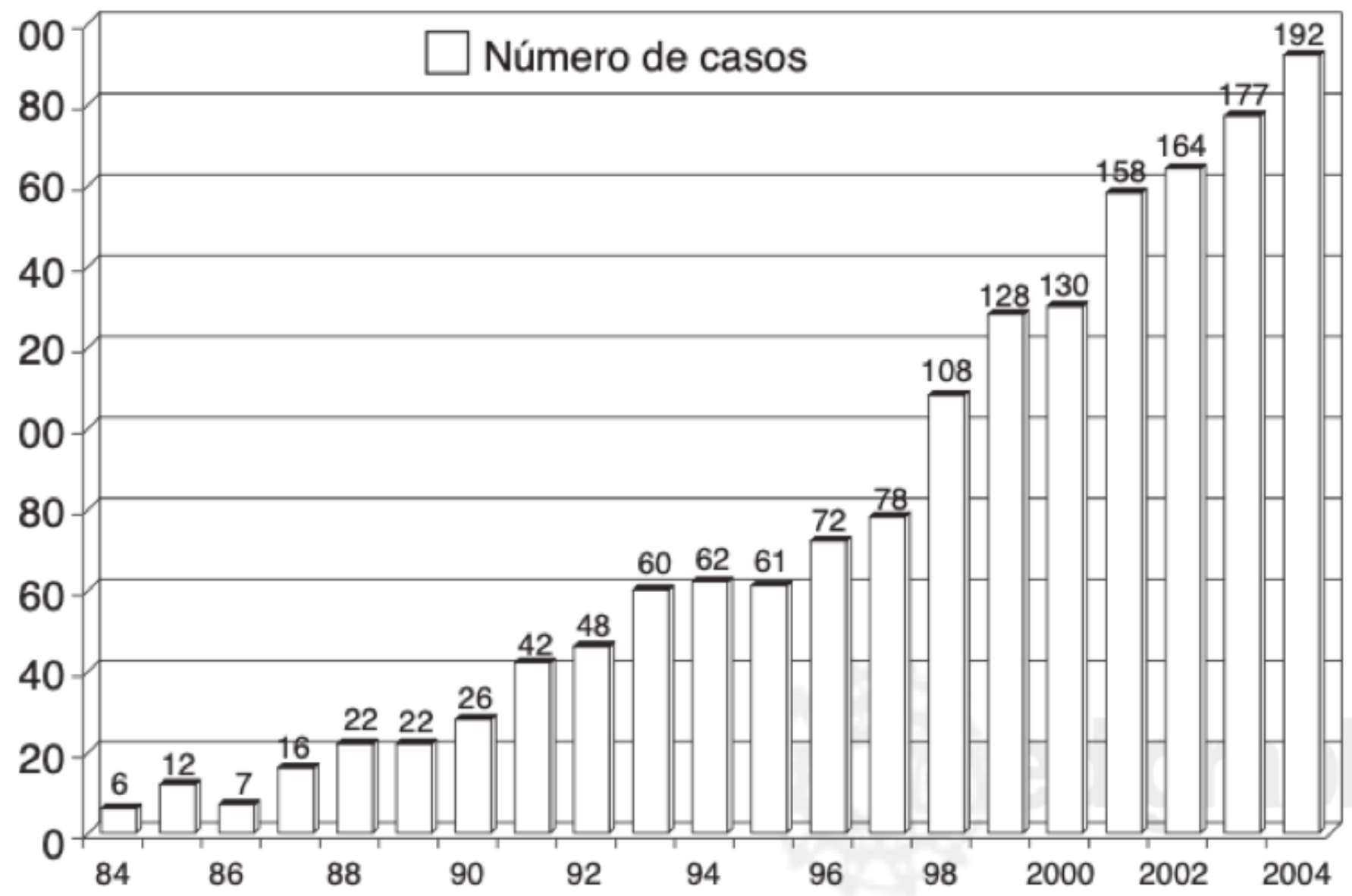

Ya teniendo en cuenta las bases de esta patología, se puede pasar al siguiente paso el cual sería su abordaje radiológico.

En general la primer área de atención donde consultarán de primera instancia los pacientes portadores de esta enfermedad es el servicio de Emergencias y por medio de la utilización de Rayos X, sin embargo no es posible hacer un diagnóstico preciso con solo esta imagen ya que según ha sido estudiado por muchos autores, el método de elección va a depender de la localización del aneurisma. Para esto se va a encontrar la siguiente clasificación: 9 


\section{CIENCIA\&SALLD}

\section{Fig. 2 Clasificación Aneurisma Aórtico}

Teniendo en cuenta la imagen anterior, se tiene una base sobre la cual se puede construir la idea de lo que está pasando al paciente y así poder abordarlo de una manera más integral y al mismo tiempo más dirigida; aunque saber la teoría no lo es todo para hacer el diagnóstico definitivo. Lo esencial para un diagnóstico correcto es: 10

- Resonancia magnética, si se encuentra disponible, es generalmente mejor para imágenes de aorta ascendente

- TAC con medio de contraste se puede usar para la imagen de arco aórtico y aorta descendente

- Ultrasonido transesofágico, si está disponible, especialmente para raíz y porción ascendente de aorta

- Angiografía

Fig.3 Aneurisma Aorta Abdominal

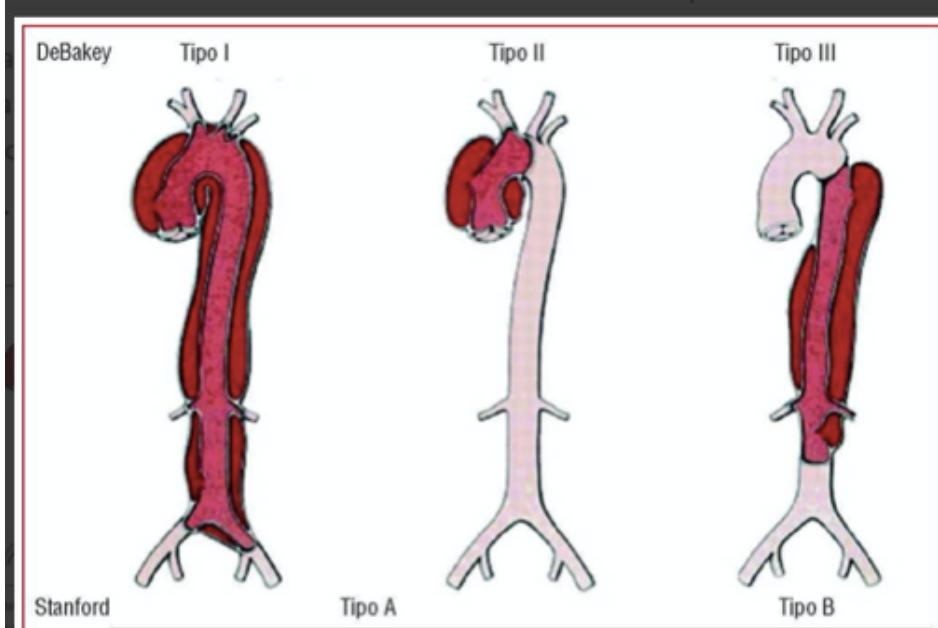

DeBakey

Tipo I. Se origina en la aorta ascendente, se propaga al menos hasta el arco de la aorta y, a menudo, más allá, en dirección distal.

Tipo II. Se origina y permanece en la aorta ascendente.

Tipo III. Se origina en la aorta descendente y se extiende en dirección distal por dicha arteria 0 , en raras ocasiones, de forma retrograda hasta el arco aórtico y la aorta ascendente. Stanford

Tipo A. Todas las disecciones afectan a la aorta ascendente, sea cual sea el sitio de origen.

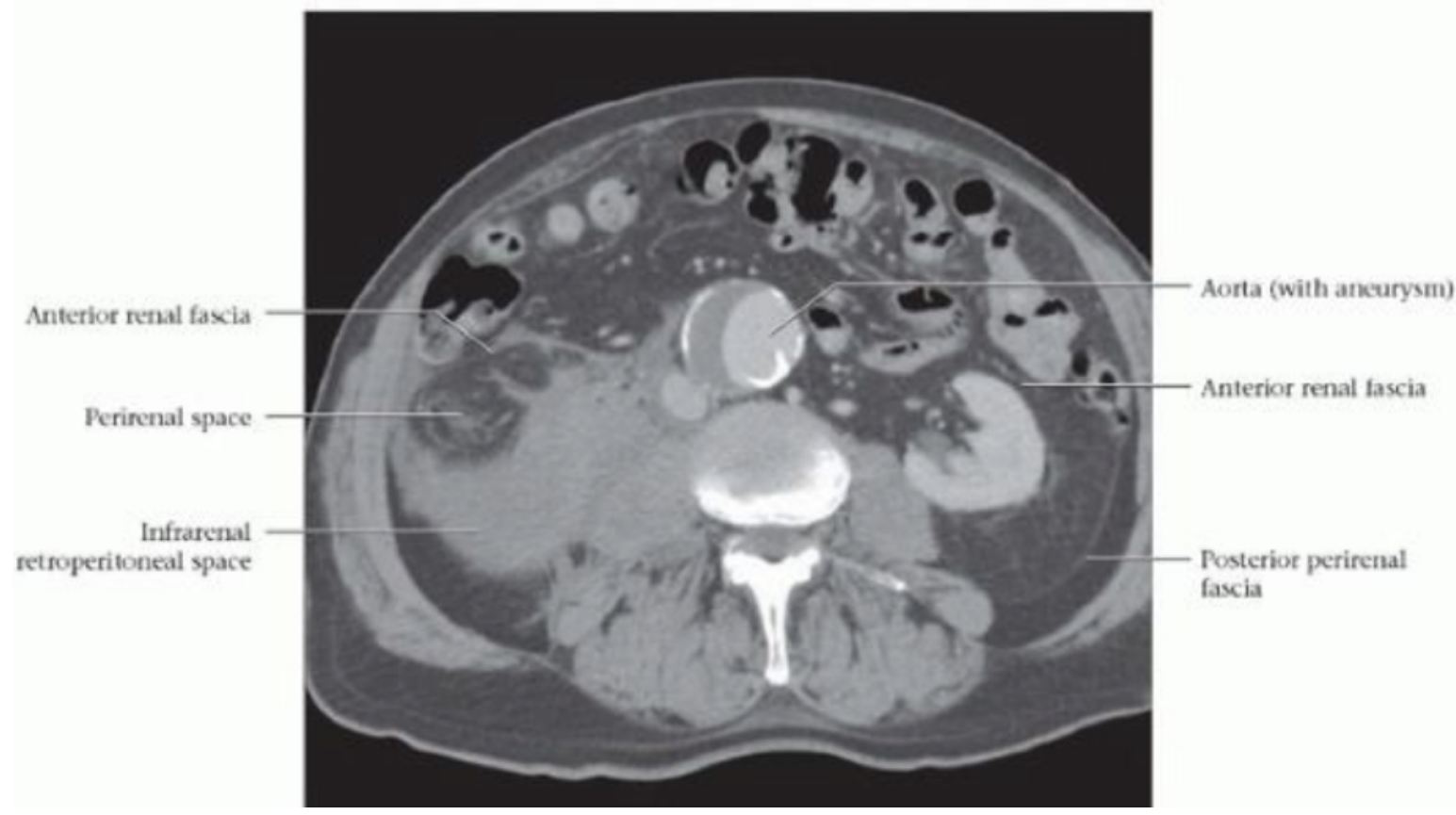

A pesar de ser una imagen de aorta abdominal, se puede apreciar la forma que toma la patología y cómo se observaría desde otro punto de vista. 11 También sirve para recalcar el hecho sobre la posibilidad aún mayor que se presente en otras localizaciones más allá de la aorta torácica. En este paciente en particular los hallazgos clínicos son diversos a los pacientes que se abordan con aneurisma torácico, según se explicará posteriormente 


\section{CIENCIA\&SALLD}

En la mayoría de centros hospitalarios, como se explicó anteriormente, se aborda principalmente este tipo de patología en el servicio de Emergencias, sin embargo un pronto diagnóstico desde la anamnesis en consulta externa podría marcar la diferencia. En el caso de diagnóstico en primera instancia como emergencia, es utilizado debido a su facilidad de acceso la radiografía de tórax, con lo cual se puede identificar el ensanchamiento mediastinal, que aunque al principio no es tan simple de ver, si se sospecha la patología es algo que ciertamente se debe buscar.

Fig. 4 Aneurisma Aórtico en Radiografía PA de Tórax

Si bien es cierto, en este caso en particular es muy evidente la patología, o al menos la anormalidad, hay que tener en cuenta que no siempre se va a

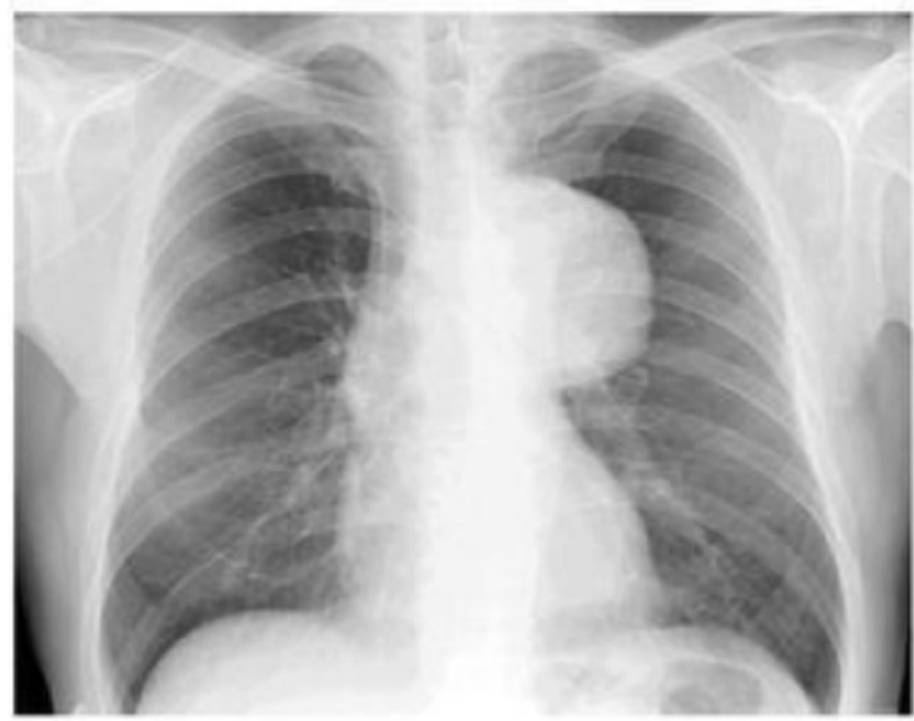
ver de esta manera, sino que puede ser un hallazgo más sutil. 12

Posterior a la radiografía, con los recursos necesarios, en caso de sospecha de un aneurisma, se debe descartar una disección, ya que esto contraindicaría el aortograma por el riesgo de ruptura. En caso de haber sido demostrado mediante clínica e imágenes que no se trata de una disección, se preferiría adicional a la angiografía como se mencionó previamente la TAC, en la cual se puede ver de diversas maneras dependiendo de la proyección; como recordatorio I

Fig. 5 Aneurisma Torácico en TAC

En la figura anterior 14 , se puede apreciar una tomografía con visualización axial demostrando el aneurisma aórtico importante, incluyendo las medidas. Por esto, la TAC es una de las mejores herramientas al momento de la evaluación diagnóstica por imágenes. Aunque no es necesariamente el médico tratante quien interpretará la TAC, sino el médico radiólogo, de igual manera es importante conocer medidas y anormalidades para dar un tratamiento más oportuno.
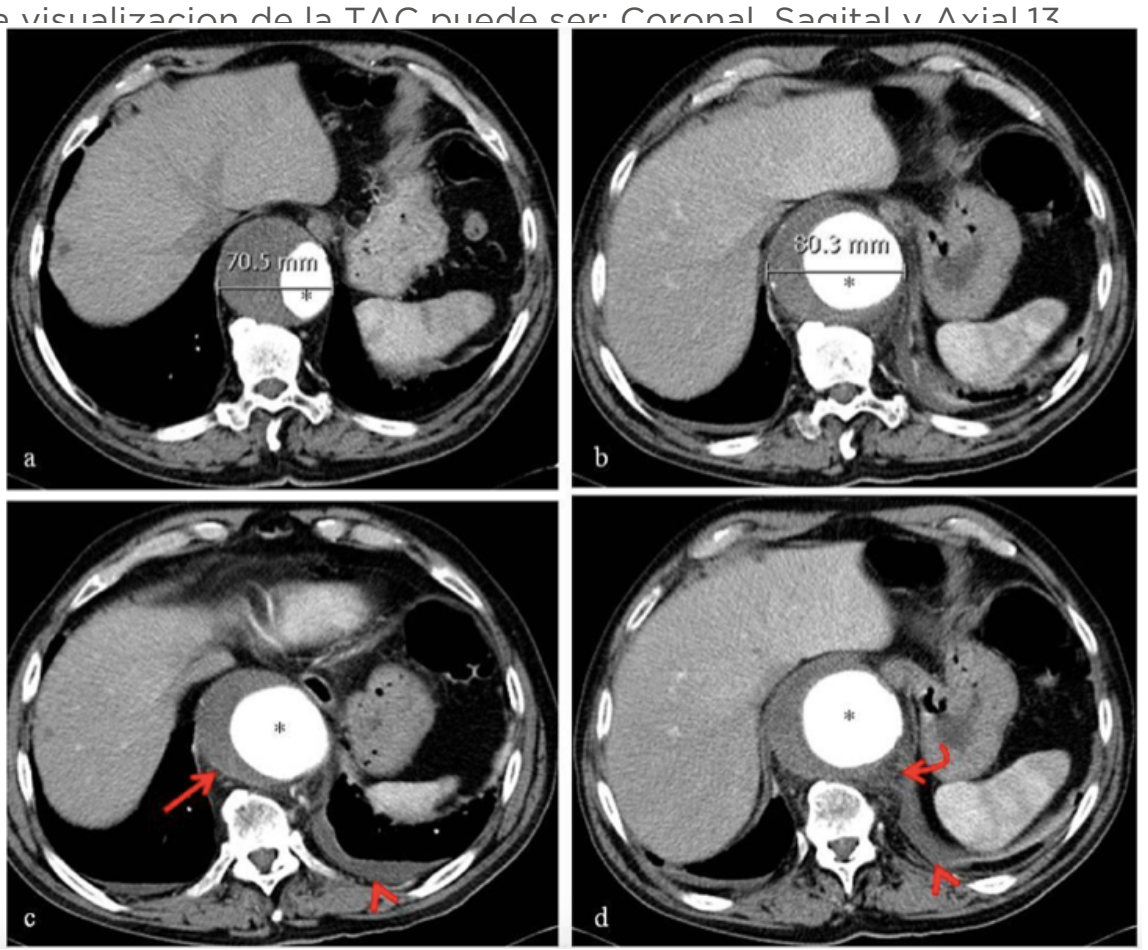


\section{CIENCIA \&SALUD}

Fig. 6 Aneurisma de Aorta Torácica vista Sagital

En la imagen previa15 se puede observar en una vista longitudinal o sagital, el aneurisma aórtico, adecuadamente señalado. Esta es una apariencia clásica en esta incidencia, la cual en momentos de duda, donde no se logre evidenciar adecuadamente con una sola proyección, se recomienda aplicar las otras formas ya descritas para tener una idea más clara de la patología.

Por otro lado se encuentra la angiografía junto con las reconstrucciones 3D, posibles por los avances tecnológicos con la TAC. La angiografía es un recurso que a pesar de producir mucha irradiacion, da resultados muy específicos y sensibles. En el caso de los aneurismas, se realiza la infusión del medio de contraste de forma intravenosa y se toman las imágenes posteriormente. Siendo este de los métodos más específicos para documentar, medir y dar seguimiento a los aneurismas. Además amplia utilización para la medición y la colocación

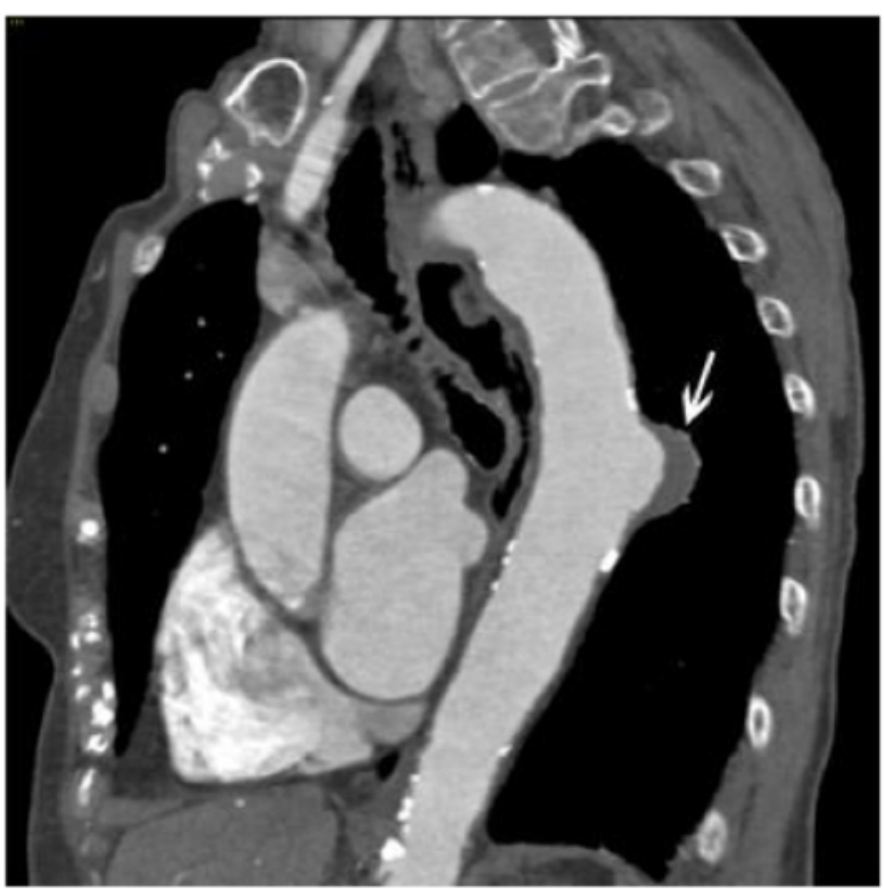
de endoprótesis.

En la figura siguiente se describe de una manera gráfica lo que se ha discutido previamente:

Fig. 7 Aneurisma Abdominal con medio de Contraste 16

Otra de las herramientas con las que se cuenta es el aortograma, el cual, de igual manera necesita un medio de contraste, se da la visualización del mismo en tiempo real, con enfoque directo a la aorta sin mostrar más estructuras.
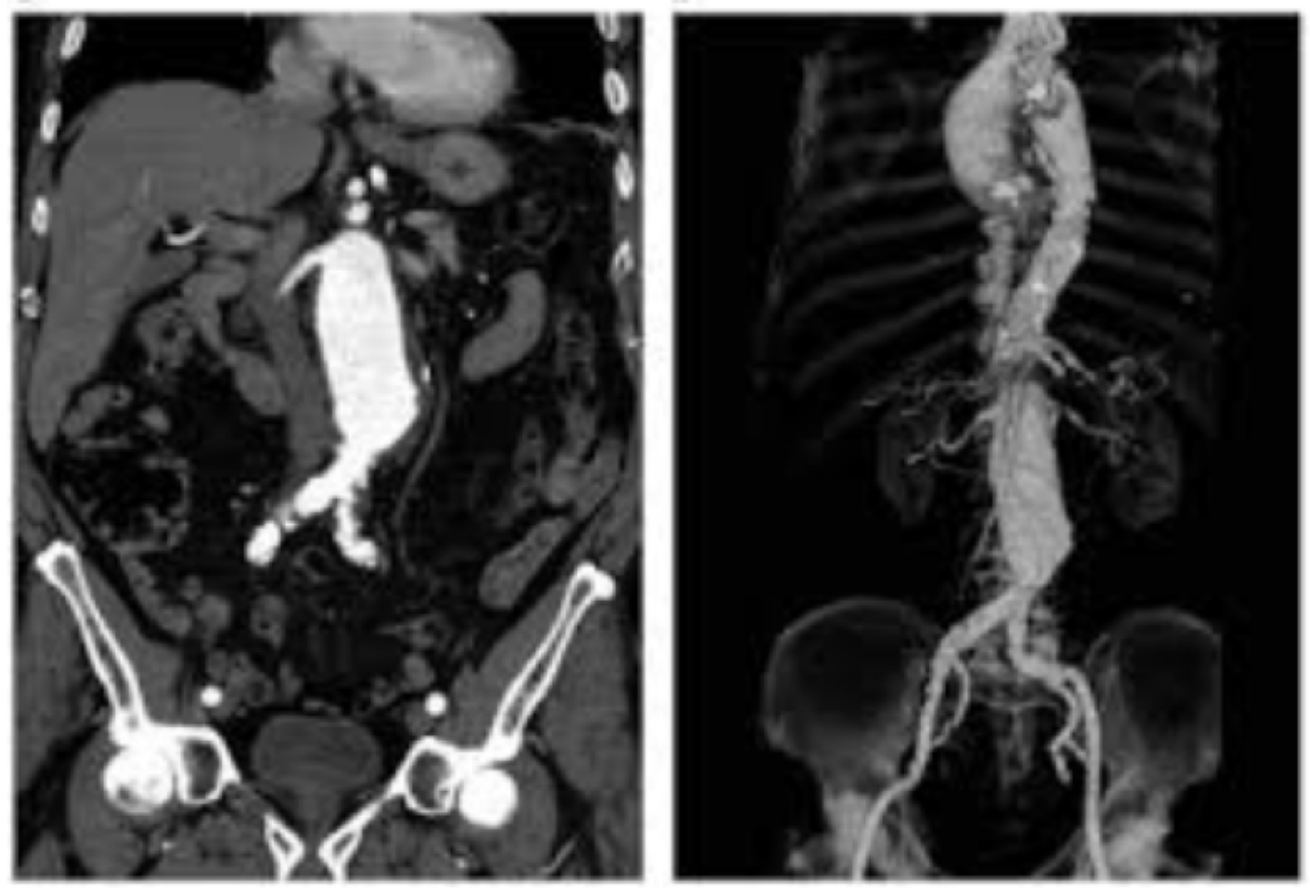


\section{CIENCIA\&SALUD}

\section{Fig.8 Aortograma normal 17}

Como se puede demostrar en la imagen, se logra ver el recorrido del contraste, además de una anatomía muy exacta, por lo cual hay mejores resultados en el momento de la evaluación del paciente.

Este último método de estudio es más complicado debido no solo a la radiación y a la experiencia del médico para realizar el estudio de manera adecuada y al mismo tiempo distinguir diversas patologías, sino que también es un recurso no siempre disponible debido al costo o por no contar con la posibilidad de un profesional que pueda realizar el procedimiento.

\section{CONCLUSIÓN}

Después de conocer un poco más sobre la enfermedad, sin entrar a fondo en las causas, agravantes o tratamientos, se puede hacer uso de varios tipos de ayuda imagenológica para confirmar la sospecha del diagnóstico.

Ya fueron abordadas diferentes proyecciones y presentaciones que pueden tener los aneurismas. Sin embargo, al momento de solicitar una evaluación radiológica, conforme a lo estudiado anteriormente, las mejores maneras de detectar la patología son la TAC y la angiografía, aunque también se pueden observar en estudios más simples como una radiografía.

\section{Conflictos de interés:}

Ninguno

\section{Fuente de financiación:}

Ninguna

\section{REFERENCIAS BIBLIOGRÁFICAS}

1. Johnston K, Rutherford R, Tilson M et al: Sugested standars for reporting on arterial aneurysms. J Vasc Surg 1991; 13: 444-50.

2. Rev. Chilena de Cirugia. Vol 56 - No 5, Octubre 2004.

3. HERRERA M, ALCÁNTARA M, LOMELÍ C, MENDOZA C, AGUIRRE C, LORENZO J, et al. Penetrating atherosclerotic ulcer of the thoracic aorta: endovascular treatment, hybrid or surgery. A review. Arch Cardiol Mex. 2009; 79: 140-6.

4. Gomez Leal (2017), Aneurismas.com.mx, Aneurisma Aorta Torácica, https://aneurismas.com.mx/ aneurisma-aorta-toracica.html

5. HIRATZKA L, BAKRIS G, BECKMAN J, BERSIN R, CARR V, CASEY D, et al. 2010 ACCF/AHA/AATS/ ACR/ASA/SCA/SCAI/SIR/STS/SVM guidelines for the diagnosis and management of patients with Thoracic Aortic Disease: a report of the American College of Cardiology Foundation/American Heart Association Task Force on Practice Guidelines, American Association for Thoracic Surgery, American 


\section{CIENCIA\&SALUD}

College of Radiology, American Stroke Association, Society of Cardiovascular Anesthesiologists, Society for Cardiovascular Angiography and Interventions, Society of Interventional Radiology,

Society of Thoracic Surgeons, and Society for Vascular Medicine. Circulation. 2010; 121: e266-369.

6. Kumar V, Abbas AK, Aster JC. Robbins y Cotran Patolog a estructural y funcional. 9th. Barcelona: Elsevier; 2015.

7. Martinez Hernandez (2006), Medigraphic, los aneurismas de la aorta tor cica y su enfoque terapeutico, https://www.medigraphic.com/pdfs/archi/ac-2006/acs062o.pdf

8. O'ROUKE MF, AVOLIO AP, NICHOLS WW: Left- ventricular-systemic arterial coupling in humans and strategies to improve coupling in desease states, in Yin FCP (ed): Ventricular/Vascular Coupling: Clinical, Physiological and Engineering Aspects. New York, Springer-Verlag, 1987.

9. Hüseyin Ince, Nienaber (2007), Revista Espa ola de Cardiolog a, Tratamiento de los Sindromes aorticos agudos, Vol 60. Num 5. P ginas $526-541$

10. William Herring, Learning Radiology, Recognizing the basics, 3 edici n, Pensylvania, Elsevier, 2016. 11. Federle, Diagnostic and Surgical Imaging Anatomy, 1 edici n, Amirsys, 2012 12. Herrera - Gomar (2009), Archivos de Cardiologia de Mexico, Elsevier, Aneurisma de aorta tor cica por Icera ateroscler tica penetrante: tratamiento endovascular, h brido o cirug a. Revisi n, vol 79, Numero 2.

13. Weir - Abrahams, Atlas de Anatomia Humana por tecnicas de imagen, Cuarta Edicion, Barcelona, Elsevier, 2011

14. Perez de Los Rios (2017), Sociedad espa ola de Radiologia de Urgencias, Signos de inminente rutura en un aneurisma de aorta toracoabdominal, http://serau.org/2017/05/signos-de-inminenteroturaen-un-aneurisma-de-aorta-toracoabdominal/

15. Farber - Ahmad (2019), Manual MSD, Aneurismas de la Aorta Toracica, https://www.msdmanuals. com/es/professional/trastornos-cardiovasculares/enfermedades-de-la-aorta-y-sus-ramas/ aneurismas-de-la-aorta-tor cica

16. Diagnostico Especializado (2016), Diagnostico especializado por Imagen, Aneurisma de la Aorta Toracica, https://www.deidiagnostico.com/aneurisma-de-la-aorta-toracica/

17. Esperon, Colegio Argentino de Cardioangiologos intervencionistas, Aortograma Toracico, http:// caci.org.ar/post/aortograma-toracico 